\title{
TOWARDS COMPLETE PHOTONIC BAND GAP STRUCTURES BELOW INFRARED WAVELENGTHS
}

\author{
Alexander Moroz ${ }^{1}$ \\ Debye Institute, Utrecht University, \\ P.O. Box 80000, 3508 TA Utrecht, The Netherlands
}

\section{INTRODUCTION}

Photonic crystals are structures with a periodically modulated dielectric constant. In analogy to the case of an electron moving in a periodic potential, certain photon frequencies can become forbidden, independent of photon polarization and the direction of propagation - a complete photonic bandgap (CPBG) [1, 2]. As early as 1975, photonic crystals with such a gap have been shown to offer the possibility of controlling the spontaneous emission of embedded atoms and molecules in volumes much greater than the emission wavelength [3] and, later on, to be an important ingredient in a variety of technological applications [4]. However, as yet no two- (2D) and three-dimensional (3D) photonic crystals are available with complete bandgaps below infrared (IR) wavelengths and fabrication of photonic crystals with such a gap poses a significant technological challenge already in the near-IR $[5,6]$. One faces the extreme difficulty in satisfying combined requirements on the dielectric contrast and the modulation (the total number and the length of periodicity steps). In order to achieve a CPBG below the IR wavelengths, the modulation is supposed to be on the scale of optical wavelengths or even shorter and, as for any CPBG structure, must be achieved with roughly ten periodicity steps in each direction. This task is currently beyond the reach of reactive ion and chemical etching techniques even for 2D structures, because the hole filling fraction must be rather high and the etching must be deep enough [7]. (See, however, [8] for a recent progress using holographic techniques.) Fortunately, in 3D, such a modulation occurs naturally in colloidal crystals formed by monodisperse colloidal suspensions of microspheres. The latter are known to self-assemble into 3D crystals with excellent long-range periodicity on the optical scale [9], removing the need for complex and costly microfabrication. Colloidal systems of microspheres crystalize either in a face-centeredcubic (fcc) or (for small sphere filling fraction) in a body-centered-cubic (bcc) lattice [9]. Since larger sphere filling fractions favour opening of larger gaps, simple fcc structures

\footnotetext{
${ }^{1}$ www.amolf.nl/research/photonic_materials_theory
} 
The outline of the contributions is as follows. First, we shall concentrate on the 3D photonic structures. In Sec. 2 we discuss photonic properties of purely dielectric fcc structures of homogeneous and coated spheres. In Sec. 3 we review our recent work $[10,11,12]$ which shows a way to avoid the restrictions on the critical dielectric contrast required for the formation of a CPBG and discuss recent experimental progress towards fabrication of a CPBG structure below IR wavelengths. In Sec. 4 we discuss the application of ideas, presented in Sec. 3, to lower dimensional photonic structures. Sec. 5 deals with nonlinear properties of metallo-dielectric structures. In Sec. 6 we review a few approaches to fabricate the proposed structures, and, in Sec. 7 we give our conclusions and outlook for future investigations.

\section{SELF-ORGANIZING COLLOIDAL STRUCTURES}

Self-organization of small colloidal particles into regular crystals on an optical scale has been known for a long time and occurs, for example, in natural opals. Indeed, the origin of the nice colours of opals is Bragg scattering. However, the crystalline order by itself is not enough to open a CPBG. Let $\varepsilon_{h}$ and $\varepsilon_{s}$ be the host and sphere dielectric constant, respectively. If $\varepsilon_{s}$ is less than $\varepsilon_{h}$, the so-called "air spheres" case, calculations show that a CPBG can open in a simple fcc structure [13], provided the dielectric contrast $\delta=\max \left(\varepsilon_{h} / \varepsilon_{s}, \varepsilon_{s} / \varepsilon_{h}\right) \gtrsim 8[14,15]$. This puts a large constraint on the materials choice which is yet to be overcome. Motivated by the experimental progress, we have investigated if it is possible to avoid the constraint on the dielectric contrast by making the scatterers more complex, for instance, by using coating spheres [15]. For the so-called stop gap (a photonic band gap in a given direction of propagation) in the (111) crystal direction, which corresponds to the L point of the Brillouin zone, the results are rather interesting. Imagine, we can fabricate fcc photonic crystals of spheres with different respective refractive indices, say $n_{1}$ and $n_{2}<n_{1}$, with the sphere filling fraction and the host refractive index being the same in both cases. Let us assume that the homogeneous spheres can be substituted by coated spheres with the core and shell refractive indices $n_{1}$ and $n_{2}$, respectively. Then the L-gap width can increase by as much as $50 \%$ relative to the larger of the L-gap widths of the two fcc photonic crystals of homogeneous spheres. Apart from larger L-gap, a suitable coating can be used to manipulate optical properties of particles in a controlled way. Using this way we can tune properties of the photonic structures which the core-shell spheres form. Unfortunately, a coating does not affect much the critical dielectric contrast necessary for the formation of a CPBG $[15,16]$. One way to avoid the restriction on the dielectric contrast is to make a radical departure from purely dielectric structures.

\section{COLLOIDAL STRUCTURES OF METALLIC SPHERES}

Development in photonic crystals has been partly stimulated by the lack of good metallic mirrors below the IR wavelengths where a typical metal absorption increases (see Figure 1). By some strange turn, a promising new route to achieve a CPBG below the IR wavelengths is to use scatterers with a negative dielectric constant $[10,11,12]$, which is usually associated with metals. For example, let us take the Drude-like dielectric 
absorption can be significantly curbed by using a periodic silver-silica layered structure compared to that of bulk silver. Surprisingly enough, the silver layer thickness in an optimized configuration is $\approx 10 \mathrm{~nm}$ for all wavelengths considered. It turns out that an optimized configuration has always the maximum allowed number $(=25)$ of unit cells, which provides a numerical justification of the photonic crystal concept.

Generally, in the presence of an absorption, eigenfrequencies $\omega$ turn into complex resonances [20]. The absorption affects a band gap in Re $\omega$ in several ways. It pushes band edges down and, mostly, increases the relative gap width $g_{w}$ (gap width to the midgap frequency ratio), sometimes by as much as $50 \%$ (although, in some cases it can also lead to a decrease in $g_{w}$ ) [21] . A band gap in Re $\omega$ must not necessarily imply a very high reflectivity. A study of finite 1D layered structures reveals that, in the presence of a strong absorption, the reflectivity at the gap center can, for instance, saturate at $60 \%$ or less, depending on a single bilayer, or, a single unit cell absorption [21]. The latter yields a lower bound on the absorption of a composite structure. The reflectivity at the gap center is usually more than twice that for frequencies within a band, almost exclusively as a result of decreased absorption within the gap which approaches that of a single bilayer. In both cases transmissions are small. If $T$ denotes the transmission for frequencies within a band, then the transmission within a gap is typically $T^{2}$ [20]. The effect of absorption on the band gap is negligible (both in shifting the gap edges and in changing gap width) if and only if $\left(\operatorname{Im} \varepsilon_{s}\right) /\left(\operatorname{Re} \varepsilon_{s}\right) \leq 0.1$.

\subsection{Photonic Structures of Coated Spheres}

The exceptional band-gap properties of Drude spheres, i.e., spheres with a Drude-like dielectric function, are rather robust against coating these spheres with a semiconductor or an insulator [12]. By using a coating which can be subsequently index-matched, one can continuously tune the metallic filling fraction. However, the coating can play a much more important role. Coating can actually facilitate the preparation of photonic colloidal crystals of Drude spheres because it can (i) stabilize metallic microparticles by preventing, or, at least, by significantly reducing their oxidation; (ii) prevent aggregation of metallic particles by reducing Van der Waals forces between them. In the latter case, a coating of roughly $30 \mathrm{~nm}$ is required. Except from enlarging of some of the stop gaps (gaps in a fixed direction of propagation) by as much as 50\% [15], the coating with an optically nonlinear material can reduce the required intensity for the onset of optical bistability $[22,23]$ due to the enhancement of local fields near the surfaceplasmon resonance. Using a procedure in which fluorescent organic groups are placed inside the silica shell with $\mathrm{nm}$ control over the radial position [24] makes it, in principle, possible to perform a precise position-dependent testing of the spontaneous emission within such a photonic crystal. Last but not least, using a semiconductor coating may allow a matching of the photonic and electronic bandgaps, which is important for many applications involving photonic crystals [4]. Our results show that for coated Drude microspheres with a coating width $l_{c} / \lambda_{p} \leq 10 \%$ (up to $l_{c}=30 \mathrm{~nm}$ for $\lambda_{p}=328 \mathrm{~nm}$ which corresponds to silver) and the coating and host refractive indices $n_{c}$ and $n_{h}$, respectively, between 1 and 1.47, one can always find a sphere radius $r_{s}$ such that the relative gap width $g_{w}$ is larger than $5 \%$ and, in some cases, $g_{w}$ can even exceed $9 \%$ [12]. This provides a sufficiently large margin for gap-edge distortions due to omnipresent imperfections and impurities to allow both technological and experimental applications involving the proposed structures. Using different coatings and by changing the refractive index $n_{h}$ of 

to whatever frequency within a nonabsorptive window $\left(0.6 \omega_{p} \leq \omega \leq 1.1 \omega_{p}\right.$ for silver $[17])$.

It is important to realize that, because the metallic core size parameter $x=2 \pi r_{c} / \lambda$ satisfies $x \geq 3.4$ for all wavelengths within a CPBG for all CPBG's considered here in the frequency region $0.55 \omega_{p} \leq \omega \leq 1.1 \omega_{p}$, the absorption is still dominated by bulk properties, i.e., can be negligible [17], since the plasmon-induced absorption becomes relevant only for particle sizes much smaller than the wavelength [25].

\section{TWO-DIMENSIONAL PHOTONIC STRUCTURES}

For many technological applications it is enough to achieve a photonic bandgap (PBG) for in-plane propagation and, for applications involving highly polarized light sources, it can be sufficient to obtain a PBG for a single polarization only (Note that for in-plane propagation, the two photon polarizations do not mix and Maxwell's equations reduce to two scalar equations, one for each polarization.). Numerous applications have been suggested involving 2D photonic structures, i.e., new designs for light-emitting diodes [26], polarizers [27], high transmission through sharp bends [28], efficient bandpass filters, channel drop filters, and, in one-dimension (1D), waveguide crossing without cross-talk [29]. For such structures, only an in-plane CPBG can ensure light propagation control whatever the in-plane light propagation. Fortunately, many of the ideas discussed for $3 \mathrm{D}$ photonic structures also apply to $2 \mathrm{D}$ metallo-dielectric structures. For square and triangular lattices of Drude cylinders, i.e., cylinders with a Drude-like behaviour of their dielectric function, we have showed that such systems can possess complete in-plane CPBG's below IR wavelengths [10]. Of the two geometries, the optimal one for ideal Drude-like behaviour is a square lattice, whereas for Drude-like behaviour in silver, using experimental data [17], the optimal geometry is a triangular lattice [10]. If the lattice spacing is tuned to a characteristic plasma wavelength, several CPBG's open in the spectrum and their relative gap width can be as large as $36.9 \%$ (9.9\% in a nonabsorptive window) even if the host dielectric constant $\varepsilon_{h}=1$ [10].

The observed magnitude and robustness of the in-plane CPBG of the metallodielectric structures allows one to speculate that an inclusion of metallic (silver) wires could boost the performance of the photonic crystal fibre designed by Knight et al. [30]. The photonic crystal fibre [30] is a 2D photonic periodic arrangement of thin cylindrical glass fibres where the light is sent along the cylinder axis. In lateral directions, the localization of light is achieved in complete analogy to the case of electrons: by introducing a defect at the center of the photonic crystal fibre, for instance, by omitting one cylinder such that it induces a transversally localized mode with frequency within a $2 \mathrm{D}$ CPBG. The light can then propagate with that frequency along the cylinder axis even if the core of the photonic crystal fibre is air and if cladding has a higher refractive index.

\section{NONLINEAR PROPERTIES OF METALLO-DIELECTRIC STRUC- TURES}

There are many potentially interesting applications involving photonic crystals with nonlinear components, for instance, as optical limiters, optical switches, optical diodes, 
billiy 22 . The with sufficiently high optical nonlinearities. For example, optically nonlinear Bragg diffracting nanosecond optical switches have been fabricated by doping of polymer particles with an absorptive dye. However, its switching efficiency has been only $\approx 2 \%$ [31].

Metals have been known to possess one of the largest nonlinear susceptibilities. Unfortunately, due to a strong metallic reflection, the light was reflected back before it could have ever experienced the metallic nonlinearity. This changes dramatically if metallic components are arranged periodically in space. It has been demonstrated in 1D that the periodicity makes metals transparent [32], thereby allowing the light to access the metallic nonlinearity [33]. This situation should not change qualitatively for 2D and 3D periodic structures. Therefore, we expect metallo-dielectric structures to be promising candidates for many nonlinear applications which can be experimentally studied in the presence of a CPBG.

\section{EXPERIMENTAL REALIZATION}

Metallo-dielectric structures can have a significant commercial impact due to their exceptional properties. We list only a few of them:

- They can yield sizeable CPBG. Depending on the scale and chosen material, a CPBG can be opened anywhere in the range from radiowaves [34] down to ultraviolet wavelengths, making the crystals an excellent template for multiplexing, waveguiding, and optical chips.

- Since metals are known to possess large nonlinear susceptibilities, the photonic crystals are inherently nonlinear, i.e., the crystals are promising candidates for various nonlinear applications.

- By applying an electric field one can switch reverseably in ms from an fcc colloidal crystal to a body-centered-tetragonal crystal: a so-called martensitic transition $[35,36,37]$. Hence, the crystals are also promising candidates for the CPBG structures with switchable bandgaps.

- Their properties can be tuned, in an irreversible way, by an ion irradiation which induces a plastic deformation of colloidal particles [38].

- A combination of a suitable coating, doping, and the presence of a metallic component allows for large freedom in optimizing specific properties of the photonic crystals.

- A nonzero electric conductivity can be used in pumping and/or in a fabrication of a new class of displays.

The main experimental problem in fabricating the proposed photonic structures in $3 \mathrm{D}$ is to synthesize large enough spheres (e.g., with radius $r_{s} \approx 150 \mathrm{~nm}$ for silver)

to reach the threshold value $r_{s} / \lambda_{p} \gtrsim 0.9 n_{h}$, where $\lambda_{p}=2 \pi \omega_{p} / c, c$ the speed of light in a vacuum, is the plasma wavelength and $n_{h}$ is the host refractive index. (Surprisingly enough, the same threshold value $r_{c} / \lambda_{p}$, where $r_{c}$ is the Drude cylinder radius, is also required to open a complete bandgap in a 2D close-packed square lattice.) Thus, the main 
materials, down to $\mathrm{GHz}$ frequencies. Correspondingly, the proposed structures can provide $\mathrm{CPBG}$ structures from the $\mathrm{GHz}$ up to ultraviolet frequencies.

Thus far, we have only investigated the simplest geometries and scatterers, i.e., only simple lattices with a single scatterer per unit cell, and only spherical and cylindrical scatterers. Although we have shown that one can achieve the relative gap width $g_{w}$ larger than $10 \%$, one expects that the width can yet be enlarged by considering lattices with more than one scatterer per unit cell [1, 40], or, using more complicated scatterers, such as cylinders with an ellipsoidal cross section [41]. These generalizations are practically very important since such structures can be now fabricated.

Recently, a very nice alternative to periodic structures proved very successful in opening a CPBG [42]. The latter involves quasicrystals which can be devised to make the Brillouin zone more spherical (circular in 2D). A quasicrystal with metallic components may be very interesting object to investigate. Likely, many open problems and many surprises are still ahead of us.

\section{ACKNOWLEDGEMENTS}

I should like to thank my colleagues A. van Blaaderen, M.J.A. de Dood, H. van der Lem, A. Polman, A. Tip, K.P. Velikov, and G.E. Zegers for careful reading of the manuscript and useful comments. This work is part of the research program by the Stichting voor Fundamenteel Onderzoek der Materie (Foundation for Fundamental Research on Matter) which was made possible by financial support from the Nederlandse Organisatie voor Wetenschappelijk Onderzoek (Netherlands Organization for Scientific Research). 
1. K.M. Ho, C.T. Chan, and C.M. Soukoulis, Existence of photonic gap in periodic dielectric structures, Phys. Rev. Lett. 65, 3152 (1990).

2. E. Yablonovitch, T.J. Gmitter, and K.M. Leung, Photonic band structure: The face-centered-cubic case employing nonspherical atoms, Phys. Rev. Lett. 67, 2295 (1991).

3. V.P. Bykov, Spontaneous emission from a medium with a band spectrum, Sov. J. Quant. Electron. 4, 861 (1975).

4. E. Yablonovitch, Inhibited spontaneous emission in solid-state physics and electronics, Phys. Rev. Lett. 58, 2059 (1987).

5. T.F. Krauss, R.M. De La Rue, and S. Brandt, Two-dimensional photonic-bandgap structures operating at near-infrared wavelengths, Nature 383, 699 (1996).

6. S.Y. Lin et al., A three-dimensional photonic crystal operating at infrared wavelengths, Nature 394, 251 (1998).

7. T.F. Krauss and R.M. De La Rue, Photonic crystals in the optical regime - past, present and future, Prog. Quant. Electronics 23, 51 (1999).

8. M. Campbell, D.N. Sharp, M.T. Harrison, R.G. Denning, and A.J. Turberfield, Fabrication of photonic crystals for the visible spectrum by holographic lithography, Nature 404, 53 (2000).

9. W.B. Russel, D.A. Saville, and W.R. Schowalter, Colloidal Dispersions, Cambridge University Press, Cambridge (1995).

10. H. van der Lem and A. Moroz, Towards two-dimensional complete photonic band gap structures below infrared wavelengths, J. Opt. A: Pure Appl. Opt. 2, 395 (2000).

11. A. Moroz, Three-dimensional complete photonic-bandgap structures in the visible, Phys. Rev. Lett. 83, 5274 (1999).

12. A. Moroz, Photonic crystals of coated metallic spheres, Europhys. Lett. 50, 466 (2000).

13. H.S. Sözüer, J.W. Haus, and R. Inguva, Photonic bands: convergence problem with the plane-wave method, Phys. Rev. B 45, 13962 (1992).

14. R. Biswas, M.M. Sigalas, G. Subramania, and K.-M. Ho, Photonic band gaps in colloidal systems, Phys. Rev. B 57, 3701 (1998).

15. A. Moroz and C. Sommers, Photonic band gaps of three-dimensional face-centered cubic lattices, J. Phys.: Condens. Matter 11, 997 (1999).

16. K. Busch and S. John, Photonic band gap formation in certain self-organizing systems, Phys. Rev. E 58, 3896 (1998).

17. E.D. Palik et al., Handbook of Optical Constants of Solids I, Academic Press, San Diego (1991).

18. A. Moroz, Density-of-states calculation and multiple scattering theory for photons, Phys. Rev. B 51, 2068 (1995).

19. W.Y. Zhang, X.Y. Lei, Z.L. Wang, D.G. Zheng, W.Y. Tam, C.T. Chan, and P. Sheng, Robust photonic band gap from tunable scatterers, Phys. Rev. Lett. 84, 2853 (2000).

20. A. Tip, J.-M. Combes, and A. Moroz, Band structure of absorptive photonic crystals, J. Phys. A: Math. Gen. 33, 6223 (2000).

21. A. Moroz, A. Tip, and J.-M. Combes, Absorption in periodic layered structures, Synthetic Metals 115, (2000) (to appear).

22. K.M. Leung, Optical bistability in the scattering and absorption of light from nonlinear microparticles, Phys. Rev. A 33, 2461 (1986).

23. D.S. Chemla and D.A. Miller, Mechanism for enhanced optical nonlinearities and bistability by combined dielectric-electronic confinement in semiconductor nanocrystallites, Opt. Lett. 11, 522 (1986).

24. A. van Blaaderen and A. Vrij, Synthesis and characterization of colloidal dispersions of fluorescent, monodisperse silica spheres, Langmuir 8, 2921 (1992).

25. C.F. Bohren and D.R. Huffman, Absorption and scattering of light by small particles, Wiley, New York, (1984), Chap. 9, 12.

26. S. Fan, P.R. Villeneuve, J.D. Joannopoulos, and E.F. Schubert, High extraction efficiency of spontaneous emission from slabs of photonic crystals, Phys. Rev. Lett. 78, 3294 (1997).

27. A.R. McGurn and A.A. Maradudin, Photonic band structures of two- and three-dimensional periodic metal or semiconductor arrays, Phys. Rev. B 48, 17576 (1993).

28. A. Mekis, J.C. Chen, I. Kurland, S. Fan, P.R. Villeneuve, and J.D. Joannopoulos, High transmission through sharp bends in photonic crystal waveguides, Phys. Rev. Lett. 77, 3787 (1996).

29. G. Johnson, C. Manolatou, S. Fan, P.R. Villeneuve, J.D. Joannopoulos, and H.A. Haus, Elimination of cross talk in waveguide intersections, Opt. Lett. 23, 1855 (1998).

30. J.C. Knight, J. Broeng, T.A. Birks, and P.St.J. Russell, A photonic crystal fibre, Science 282, 1476 (1998). 
32. M. Scalora, M.J. Bloemer, A.S. Pethel, J.P. Dowling, C.M. Bowden, and A.S. Manaka, Transparent, metallo-dielectric, one-dimensional, photonic band-gap structures, J. Appl. Phys. 83, 2377 (1998).

33. R.S. Bennink, Y.-K. Yoon, R.W. Boyd, and J.E. Sipe, Accessing the optical nonlinearity of metals with metal-dielectric photonic bandgap structures, Opt. Lett. 24, 1416 (1999).

34. J.B. Pendry, A.J. Holden, W.J. Stewart, and I. Youngs, Extremely low frequency plasmons in metallic mesostructures, Phys. Rev. Lett. 76, 4773 (1996).

35. A. van Blaaderen, From the de Broglie to visible wavelengths: Manipulating electrons and photons with colloids, MRS Bulletin (October) 23, 39 (1998).

36. W. Wen, N. Wang, H. Ma, Z. Lin, W.Y. Tam, C.T. Chan, and P. Sheng, Field induced structural transition in mesocrystallites, Phys. Rev. Lett. 82, 4248 (1997).

37. U. Dassanayake, S. Fraden, and A. van Blaaderen, Structure of electrorheological fluids, J. Chem. Phys. 112, 3851 (2000).

38. E. Snoeks, A. van Blaaderen, T. van Dillen, C.M. van Kats, M.L. Brongersma, and A. Polman, Colloidal ellipsoids with continuously variable shape, Adv. Materials 12, 1511 (2000).

39. D.V. Goia and E. Matijecic, Tailoring the particle size of monodispersed colloidal gold, New Journal of Chemistry 146, 139 (1999).

40. D. Cassagne, C. Jouanin, and D. Bertho, Hexagonal photonic-band-gap structures, Phys. Rev. B 53, 7134 (1996).

41. M. Qiu and S. He, Large complete band gap in two-dimensional photonic crystals with elliptic air holes, Phys. Rev. B 60, 10610 (1999).

42. M.E. Zoorob, M.D.B. Charlton, G.J. Parker, J.J. Baumberg, and M.C. Netti, Complete photonic bandgaps in 12-fold symmetric quasicrystals, Nature 404, 740 (2000). 
Fig. 1 - Reflectivity at normal incidence of bulk silver compared to that of optimized finite silver-silica stack. Each data point is an optimum over $\approx 2.9$ million different configurations examined.

Fig. 2 - Large silver spheres fabricated by K.P. Velikov and G.E. Zegers. a) Scanning electron micrograph (SEM) of dried silver particles with radius $r_{s}=349 \mathrm{~nm}$ and polydispersity $\delta_{d}=16 \%$ ). Only small ordered regions are observed. b) Confocal image of the bottom layer of a charge stabilized crystal of silver particles $\left(r_{s}=320 \mathrm{~nm}, \delta_{d}<17 \%\right)$ in water. Large single crystals with a pitch of $1 \mu \mathrm{m}$ are observed, as shown in the inset. 
[1] K.M. Ho, C.T. Chan, and C.M. Soukoulis, Existence of photonic gap in periodic dielectric structures, Phys. Rev. Lett. 65, 3152 (1990).

[2] E. Yablonovitch, T.J. Gmitter, and K.M. Leung, Photonic band structure: The face-centeredcubic case employing nonspherical atoms, Phys. Rev. Lett. 67, 2295 (1991).

[3] V.P. Bykov, Spontaneous emission from a medium with a band spectrum, Sov. J. Quant. Electron. 4, 861 (1975).

[4] E. Yablonovitch, Inhibited spontaneous emission in solid-state physics and electronics, Phys. Rev. Lett. 58, 2059 (1987).

[5] T.F. Krauss, R.M. De La Rue, and S. Brandt, Two-dimensional photonic-bandgap structures operating at near-infrared wavelengths, Nature 383, 699 (1996).

[6] S.Y. Lin et al., A three-dimensional photonic crystal operating at infrared wavelengths, Nature 394, 251 (1998).

[7] T.F. Krauss and R.M. De La Rue, Photonic crystals in the optical regime - past, present and future, Prog. Quant. Electronics 23, 51 (1999).

[8] M. Campbell, D.N. Sharp, M.T. Harrison, R.G. Denning, and A.J. Turberfield, Fabrication of photonic crystals for the visible spectrum by holographic lithography, Nature 404, 53 (2000).

[9] W.B. Russel, D.A. Saville, and W.R. Schowalter, Colloidal Dispersions, Cambridge University Press, Cambridge (1995).

[10] H. van der Lem and A. Moroz, Towards two-dimensional complete photonic band gap structures below infrared wavelengths, J. Opt. A: Pure Appl. Opt. 2, 395 (2000).

[11] A. Moroz, Three-dimensional complete photonic-bandgap structures in the visible, Phys. Rev. Lett. 83, 5274 (1999).

[12] A. Moroz, Photonic crystals of coated metallic spheres, Europhys. Lett. 50, 466 (2000).

[13] H.S. Sözüer, J.W. Haus, and R. Inguva, Photonic bands: convergence problem with the planewave method, Phys. Rev. B 45, 13962 (1992).

[14] R. Biswas, M.M. Sigalas, G. Subramania, and K.-M. Ho, Photonic band gaps in colloidal systems, Phys. Rev. B 57, 3701 (1998).

[15] A. Moroz and C. Sommers, Photonic band gaps of three-dimensional face-centered cubic lattices, J. Phys.: Condens. Matter 11, 997 (1999).

[16] K. Busch and S. John, Photonic band gap formation in certain self-organizing systems, Phys. Rev. E 58, 3896 (1998).

[17] E.D. Palik et al., Handbook of Optical Constants of Solids I, Academic Press, San Diego (1991).

[18] A. Moroz, Density-of-states calculation and multiple scattering theory for photons, Phys. Rev. B 51, 2068 (1995).

[19] W.Y. Zhang, X.Y. Lei, Z.L. Wang, D.G. Zheng, W.Y. Tam, C.T. Chan, and P. Sheng, Robust photonic band gap from tunable scatterers, Phys. Rev. Lett. 84, 2853 (2000).

[20] A. Tip, J.-M. Combes, and A. Moroz, Band structure of absorptive photonic crystals, J. Phys. A: Math. Gen. 33, 6223 (2000).

[21] A. Moroz, A. Tip, and J.-M. Combes, Absorption in periodic layered structures, Synthetic Metals 115, (2000) (to appear).

[22] K.M. Leung, Optical bistability in the scattering and absorption of light from nonlinear microparticles, Phys. Rev. A 33, 2461 (1986).

[23] D.S. Chemla and D.A. Miller, Mechanism for enhanced optical nonlinearities and bistability by combined dielectric-electronic confinement in semiconductor nanocrystallites, Opt. Lett. 11, 522 (1986).

[24] A. van Blaaderen and A. Vrij, Synthesis and characterization of colloidal dispersions of fluorescent, monodisperse silica spheres, Langmuir 8, 2921 (1992). 
[26] S. Fan, P.R. Villeneuve, J.D. Joannopoulos, and E.F. Schubert, High extraction efficiency of spontaneous emission from slabs of photonic crystals, Phys. Rev. Lett. 78, 3294 (1997).

[27] A.R. McGurn and A.A. Maradudin, Photonic band structures of two- and three-dimensional periodic metal or semiconductor arrays, Phys. Rev. B 48, 17576 (1993).

[28] A. Mekis, J.C. Chen, I. Kurland, S. Fan, P.R. Villeneuve, and J.D. Joannopoulos, High transmission through sharp bends in photonic crystal waveguides, Phys. Rev. Lett. 77, 3787 (1996).

[29] G. Johnson, C. Manolatou, S. Fan, P.R. Villeneuve, J.D. Joannopoulos, and H.A. Haus, Elimination of cross talk in waveguide intersections, Opt. Lett. 23, 1855 (1998).

[30] J.C. Knight, J. Broeng, T.A. Birks, and P.St.J. Russell, A photonic crystal fibre, Science 282, 1476 (1998).

[31] G. Pan, R. Kesavamoorthy, and S.A. Asher, Optically nonlinear Bragg diffracting nanosecond optical switches, Phys. Rev. Lett. 78, 3860 (1997).

[32] M. Scalora, M.J. Bloemer, A.S. Pethel, J.P. Dowling, C.M. Bowden, and A.S. Manaka, Transparent, metallo-dielectric, one-dimensional, photonic band-gap structures, J. Appl. Phys. 83, 2377 (1998).

[33] R.S. Bennink, Y.-K. Yoon, R.W. Boyd, and J.E. Sipe, Accessing the optical nonlinearity of metals with metal-dielectric photonic bandgap structures, Opt. Lett. 24, 1416 (1999).

[34] J.B. Pendry, A.J. Holden, W.J. Stewart, and I. Youngs, Extremely low frequency plasmons in metallic mesostructures, Phys. Rev. Lett. 76, 4773 (1996).

[35] A. van Blaaderen, From the de Broglie to visible wavelengths: Manipulating electrons and photons with colloids, MRS Bulletin (October) 23, 39 (1998).

[36] W. Wen, N. Wang, H. Ma, Z. Lin, W.Y. Tam, C.T. Chan, and P. Sheng, Field induced structural transition in mesocrystallites, Phys. Rev. Lett. 82, 4248 (1997).

[37] U. Dassanayake, S. Fraden, and A. van Blaaderen, Structure of electrorheological fluids, J. Chem. Phys. 112, 3851 (2000).

[38] E. Snoeks, A. van Blaaderen, T. van Dillen, C.M. van Kats, M.L. Brongersma, and A. Polman, Colloidal ellipsoids with continuously variable shape, Adv. Materials 12, 1511 (2000).

[39] D.V. Goia and E. Matijecic, Tailoring the particle size of monodispersed colloidal gold, New Journal of Chemistry 146, 139 (1999).

[40] D. Cassagne, C. Jouanin, and D. Bertho, Hexagonal photonic-band-gap structures, Phys. Rev. B 53, 7134 (1996).

[41] M. Qiu and S. He, Large complete band gap in two-dimensional photonic crystals with elliptic air holes, Phys. Rev. B 60, 10610 (1999).

[42] M. E. Zoorob, M. D. B. Charlton, G. J. Parker, J. J. Baumberg, and M. C. Netti, Complete photonic bandgaps in 12-fold symmetric quasicrystals, Nature 404, 740 (2000). 